\title{
Model Complex Processing Navigation Signals when they are Detected in Terms of Interfering Reflections from the Background Fluctuation Noise
}

\author{
Viktor N. Bondareva, \\ Aleksey F. Evstafiev ${ }^{b}$ and Fedor A. Evstafiev \\ ${ }^{a}$ Siberian Federal University \\ 79 Svobodny, Krasnoyarsk, 660041, Russia \\ ${ }^{b}$ Military Education and Research Centre of Military-Air Forces \\ "Military-Air Academy \\ Named After Professor N.E. Zhukovsky and Yu.A. Gagarin» \\ 54a Starykh Bolshevikov Str., Voronezh, 394064, Russia
}

Received 20.01.2016, received in revised form 14.02.2017, accepted 27.02.2017

The proposed methodology and the corresponding model coherent and noncoherent detection of navigation signals in the presence of interfering reflections from background noise oscillations. The method consists in accounting for the differences between a distorted form of the navigation signal due to interfering reflections and correlation function of noise in the output part of an RF receiver. This circumstance allows to use the integrated (dual) processing the received navigation signal when it is detected with an automatically adjustable threshold decision. It is shown that this combined detection method virtually neutralizes the interfering signal, even with significant temporal overlaps with the navigation signal.

Keywords: radio-navigation signal, integrated processing, sequential detection, non-coherent detection, combined detection method.

Citation: Bondarev V.N., Evstafiev A.F., Evstafiev F.A. Model complex processing navigation signals when they are detected in terms of interfering reflections from the background fluctuation noise, J. Sib. Fed. Univ. Eng. technol., 2017, 10(2), 142-153. DOI: 10.17516/1999-494X-2017-10-2-142-153.

(c) Siberian Federal University. All rights reserved

* Corresponding author E-mail address: lyutikovigor@mail.ru 


\title{
Модели комплексной обработки
}

\section{радионавигационных сигналов при их обнаружении \\ в условиях мешающих отражений \\ на фоне флюктуационного шума}

\author{
В.Н. Бондарев ${ }^{a}$, А.Ф. Евстафиев ${ }^{\sigma}$, Ф.А. Евстафиев ${ }^{\sigma}$ \\ ${ }^{a}$ Сибирский федеральный университет \\ Россия, 660041, Красноярск, пр. Свободный, 79 \\ ${ }^{\sigma}$ Военный учебно-научный центр Военно-воздушных сил \\ «Военно-воздушная академия \\ имени профессора Н.Е. Жуковского и Ю.А. Гагарина» \\ Россия, 394064, Воронеж, ул. Старых Большевиков, 54 а
}

\begin{abstract}
Предложен способ и соответствующие модели когерентного и некогерентного обнаружения навигаџионных радиосигналов при наличии мешающих отражений на фоне флюктуационного шума. Сущность способа заключается в учете различий между формой искаженного навигационного сигнала за счет мешающих отражений и корреляционной функции шума на выходе радиочастотной части соответствующего приемника. Данное обстоятельство позволяет использовать комплексную (двухканальную) обработку принимаемого навигационного радиосигнала при его обнаружении с автоматически управляемым порогом принятия решения. Показано, что данный комбинированный способ обнаружения практически нейтрализует мешающий радиосигнал даже при значительных временных перекрытиях его с навигаиионным сигналом.
\end{abstract}

Ключевые слова: радионавигачионный сигнал, комплексная обработка, когерентное обнаружение, некогерентное обнаружение, комбинированный способ обнаружения.

\section{Введение}

Актуальность проблемы обнаружения навигационных сигналов в радиотехнических системах (РТС) ближней навигации на основе псевдоспутников обусловлена наличием в радиоканалах так называемых мешающих сигналов, порожденных отражением радиоволн от посторонних предметов, границ раздела сред и от различных неоднородностей на трассе их распространения [1-4]. При этом мешающие сигналы представляют собой суперпозиции собственных копий сигналов, сдвинутых в общем случае хаотично по времени и частоте, которые при взаимодействии с основным сигналом вызывают его интерференционные замирания [5-7]. Если при значительном временном перекрытии мешающий сигнал окажется в противофазе с основным сигналом, то это приведет к резкому уменьшению амплитуды основного сигнала и, следовательно, к уменьшению вероятности обнаружения сигнала на фоне флюктуационного шума. А поскольку начальная фаза мешающего радиосигнала, как правило, является случайной величиной, равновероятно распределенной в интервале $[-\pi, \pi]$, то это обстоятельство в конечном итоге приведет к существенному снижению помехоустойчивости (достоверности) обнаружения основного навигационного сигнала [5].

При этом если начальная фаза мешающего радиосигнала, обусловленная его случайным временным сдвигом, изменяется медленно, то и замирания результирующего сигнала будут

$$
-143-
$$


медленными, а если начальная фаза изменяется быстро и дополнительно к этому заметно влияют дестабилизирующие факторы радиоканала, то и замирания результирующего сигнала будут быстрыми.

При медленных замираниях для повышения достоверности обнаружения сигнала необходимо использовать частотно-временной резерв радиоканала, который можно реализовать, например, путем временного или (и) частотного разнесения элементов сигнала. А при быстрых замираниях необходимо использовать специальные сигналы с расширением спектра, так называемые широкополосные шумоподобные сигналы (ШПС) с последующей их оптимальной обработкой.

Недостатки этих способов очевидны: они требуют увеличения частотно-временного ресурса радиоканала, что не всегда возможно.

Цель данного исследования - разработка способа и соответствующих моделей обработки принимаемого навигационного сигнала, обладающих повышенной помехоустойчивостью его обнаружения в условиях мешающих отражений и флюктуационного шума без увеличения энергетических и частотно-временных ресурсов навигационного радиоканала.

\section{Постановка задачи}

Рассматривается РТС ближней навигации на основе псевдоспутников, работающая в режиме приема радиосигналов в условиях мешающих отражений и флюктуационного шума, задачей которой служит установление факта наличия или отсутствия навигационного сигнала в радиоканале по соответствующему критерию, в данном случае Неймана-Пирсона. При этом мешающий радиосигнал является сигналом со случайной начальной фазой, равновероятно распределенной в интервале $[-\pi, \pi]$, и параметрами такими же, как и у сигнала навигационного с соответствующим перекрытием с ним по времени. Считается при этом, что отражения локализованы только на временной оси запаздываний и что последующие по времени мешающие сигналы не оказывают сколь-либо заметного влияния на данный навигационный сигнал в момент его отсчета и при принятии решения не учитываются. Полученная таким образом сигнально-помеховая реализация принимается на фоне флюктуационного шума с равномерной спектральной плотностью мощности в полосе частот радиоприема и с нормальным законом распределения мгновенных значений.

В результате взаимодействия с мешающим радиосигналом полезный радионавигационный сигнал на выходе согласованного фильтра искажается и существенно отличается по своей форме от формы корреляционной функции выходного флюктуационного шума согласованного фильтра соответствующего приемника. Данное обстоятельство позволяет использовать комплексную обработку радионавигационного сигнала при его обнаружении в условиях мешающих отражений на фоне флюктуационного шума.

Под комплексной обработкой навигационного сигнала при наличии мешающих отражений понимается его двухканальная обработка, в первом (основном) канале которой осуществляется согласованная фильтрация сигнала с последующим принятием решения о его наличии или отсутствии, а во втором (дополнительном) канале решение о наличии или отсутствии сигнала принимается по выходному сигналу того же согласованного фильтра, но относительно значения шума, измеренного в опорном отсчете. При этом момент опорного

$$
-144-
$$


отсчета определяется точкой пересечения огибающих основного (навигационного) и мешающего радиосигналов и при значительном их временном перекрытии находится в непосредственной близости с моментом информационного отсчета, что обусловливает высокую корреляцию флюктуационного шума между этими отсчетами. По значению шума, измеренного в опорном отсчете, устанавливается значение управляемого порога во втором (дополнительном) канале обработки. В результате итоговое решение о наличии или отсутствии сигнала принимается на основе двух частных решений по правилу: сигнал обнаруживается, если хотя бы в одном из частных каналов обработки он регистрируется (выше соответствующего порога), в противном случае принимается решение об отсутствии сигнала в радиоканале.

Принятые допущения не накладывают принципиальных ограничений на общность постановки задачи и отражают достаточно реальный режим работы навигационной РТС по обнаружению навигационного сигнала в условиях мешающих отражений и флюктуационного шума. При этом в зависимости от характера изменения начальной фазы навигационного сигнала рассматриваются модели его когерентного и некогерентного обнаружения.

\section{Когерентное обнаружение}

При когерентном обнаружении предполагается, что начальная фаза навигационного сигнала точно известна и без потери общности решаемой задачи принимается равной нулю. При этом в общем случае обнаруживаемый выходной сигнал согласованного фильтра приемника будет иметь вид

$$
u_{1}(t)=U_{1}(t) \cos \omega_{0} t, \quad 0 \leq t \leq T,
$$

где $U_{1}(t), \omega_{0}, T$ - огибающая частота и длительность навигационного радиосигнала [8].

Мешающий радиосигнал на выходе фильтра, перекрытый во времени с основным радиосигналом, будет равен

$$
u_{2}(t)=U_{2}\left[t-\left(t_{0} \pm \Delta t\right)\right] \cos \omega_{0}\left[t-\left(t_{0} \pm \Delta t\right)\right], t_{0} \pm \Delta t \leq t \leq T+t_{0} \pm \Delta t
$$

где $U_{2}(t), t_{0}, \pm \Delta t$ - соответственно огибающая мешающего радиосигнала, постоянная составляющая времени задержки и случайный временной сдвиг.

В момент отсчета при $t=0$ амплитуда сигнала, определяемого суммой сигналов (1) и (2), по которой принимается решение, будет равна

$$
\begin{aligned}
& U_{\text {pе } 3}(0, \psi)=\sqrt{U_{1}^{2}(0)+U_{2}^{2}[-T(1-\chi)]+2 U_{1}(0) U_{2}[-T(1-\chi)] \cos \left(\psi-\varphi_{0}\right)} \times \\
& \times \sin \left[\operatorname{arctg} \frac{U_{1}(0)+U_{2}[-T(1-\chi)] \cos \left(\varphi_{0}-\psi\right)}{U_{2}[-T(1-\chi)] \sin \left(\varphi_{0}-\psi\right)}\right],
\end{aligned}
$$

где $\chi=1-t_{0} / T$ - относительное перекрытие навигационного и мешающего сигналов; $\varphi_{0}=\omega_{0} t_{0}-$ постоянная начальная фаза; $\psi=\omega_{0}( \pm \Delta t)-$ случайная фаза, изменяющаяся по равновероятному закону в интервале $[-\pi, \pi]$.

С учетом (3) вероятность правильного обнаружения сигнала при заданной вероятности ложной тревоги $P_{1 F}$ в первом канале обработки, в котором используется известное одноканальное обнаружение, в наших обозначениях будет равна

$$
-145-
$$




$$
P_{1 D}(\psi)=1-\Phi\left\{\Phi^{-1}\left(1-P_{1 F}\right)-\frac{U_{\mathrm{pe3}}(0, \psi)}{\sqrt{P_{\mathrm{II}}}}\right\}
$$

где $\Phi^{-1}(\cdot)$ - функция, обратная интегралу вероятности; $P_{\text {ш }}$ мощность флюктуационного шума на выходе согласованного фильтра [8].

В этом случае порог принятия решения является постоянной величиной и определяется известным выражением [8]

$$
\Pi=\sqrt{P_{\amalg}} \Phi^{-1}\left(1-P_{F}\right) .
$$

Для нахождения вероятности правильного обнаружения сигнала во втором канале обработки необходимо определить момент опорного отсчета $\tau_{0}$ из условия равенства огибающих основного и мешающего радиосигналов:

$$
U_{1}(t)=U_{2}[t-T(1-\chi)] .
$$

В общем виде решение уравнения (6) будет следующим:

$$
\tau_{0}=F\left[T(1-\chi), \alpha_{1}, \ldots, \alpha_{n}, \beta_{1}, \ldots, \beta_{m}\right],
$$

где $F(\cdot)$ - некоторая функция, зависящая от формы сигналов; $\alpha_{1}, \ldots, \alpha_{n}$ и $\beta_{1}, \ldots, \beta_{m}$ - параметры, характеризующие форму навигационного и мешающего сигналов соответственно.

Значение $\tau_{0}$ определяется с точностью до целого числа периодов коэффициента корреляции шума $r\left(\tau_{0}\right)$ на выходе согласованного фильтра.

Остаточная мощность выходного шума согласованного фильтра в момент информационного отсчета относительно опорного будет равна [9]

$$
P_{\text {шा ост }}=P_{\text {шा }}\left[1-r^{2}\left(\tau_{0}\right)\right] .
$$

При этом остаточная амплитуда сигнала в момент принятия решения определяется выражением

$$
U_{\text {ост }}(0, \psi)=U_{\text {рез }}(0, \psi)-r\left(\tau_{0}\right) U_{\text {рез }}\left(\tau_{0}, \psi\right),
$$

где $U_{\text {рез }}(0, \psi)$ определяется выражением $(3)$, а $U_{\text {рез }}\left(\tau_{0}, \psi\right)$ - результирующая амплитуда навигационного и мешающего сигналов в опорном отсчете. Эта амплитуда определяется аналогично (3) с учетом того, что $U_{1}\left(\tau_{0}\right) \approx U_{2}\left[\tau_{0}-T(1-\chi)\right]$, а фазовые соотношения здесь сохраняются такими же, как и в (3). В результате, опуская промежуточные преобразования, получим

$$
U_{\text {peз }}\left(\tau_{0}, \psi\right)=U_{1}\left(\tau_{0}\right) \sqrt{2\left[1+\cos \left(\varphi_{0}-\psi\right)\right]} \sin \left[\operatorname{arctg} \frac{1+\cos \left(\varphi_{0}-\psi\right)}{\sin \left(\varphi_{0}-\psi\right)}\right] .
$$

С учетом (4), (8) и (10) вероятность правильного обнаружения сигнала во втором дополнительном канале обработки будет определяться следующим выражением: 


$$
P_{2 D}(\psi)=1-\Phi\left\{\Phi^{-1}\left(1-P_{2 F}\right)-\frac{U_{\mathrm{pe} 3}(0, \psi)+r\left(\tau_{0}\right) U_{\mathrm{pe} 3}\left(\tau_{0}, \psi\right)}{\sqrt{P_{\mathrm{mI}}\left[1-r^{2}\left(\tau_{0}\right)\right]}}\right\}
$$

В этом канале порог принятия решения является переменной величиной и определяется выражением

$$
\Pi\left(\tau_{0}\right)=r\left(\tau_{0}\right) u_{\mathrm{I}}\left(\tau_{0}\right)+\sqrt{P_{\mathrm{II}}\left[1-r^{2}\left(\tau_{0}\right)\right]} \Phi^{-1}\left(1-P_{F}\right),
$$

где $u_{\text {шा }}\left(\tau_{0}\right)$ - измеренное текущее значение шума в опорном отсчете [10].

С учетом (4) и (11) результирующая вероятность правильного обнаружения сигнала будет равна

$$
P_{D \text { рез }}(\psi)=1-\left[1-P_{1 D}(\psi)\right]\left[1-P_{2 D}(\psi)\right]
$$

При этом средняя результирующая вероятность правильного обнаружения по всем значениям случайной начальной фазы ч мешающего сигнала определяется выражением

$$
\bar{P}_{D \text { рез }}=\frac{1}{2 \pi} \int_{-\pi}^{\pi} P_{D \text { рез }}(\psi) d \psi .
$$

Результирующая ложная тревога в данном способе обнаружения будет иметь место в том случае, если при отсутствии сигнала помеха хотя бы в одном из каналов обработки превысит порог. При одинаковых вероятностях частной ложной тревоги в каналах обработки $P_{1 F}=P_{2 F}=P_{1,2 F}$ результирующая вероятность ложной тревоги будет равна

$$
P_{F \text { рез }}=1-\left(1-P_{1 F}\right)\left(1-P_{2 F}\right)=1-\left(1-P_{1,2 F}\right)^{2} .
$$

Таким образом, получением результирующей вероятности правильного обнаружения сигнала и результирующей вероятности ложной тревоги заканчивается анализ данного обнаружителя.

\section{Некогерентное обнаружение}

Во многих случаях начальная фаза навигационного сигнала, как и мешающего, является случайной величиной, как правило, равновероятно распределенной в интервале $[-\pi, \pi]$. В этом случае необходимо использовать двухканальное некогерентное обнаружение, при котором все операции обработки сигнально-помеховых реализаций осуществляются с их огибающими. Рассмотрим эффективность данной модели обнаружения навигационного сигнала в условиях мешающего сигнала на фоне белого шума.

Пусть обнаруживаемый выходной сигнал согласованного фильтра в общем случае имеет вид

$$
S_{1}(t)=U_{1}(t) \cos \left(\omega_{0} t-\varphi_{1}\right)
$$

где $\varphi_{1}-$ случайная начальная фаза, равновероятно распределенная в интервале $[-\pi, \pi]$.

При этом мешающий радиосигнал на выходе согласованного фильтра будет определяться выражением 


$$
S_{2}(t)=U_{2}[t-T(1-\chi)] \cos \left(\omega_{0} t-\varphi_{1}-\psi-\varphi_{0}\right)
$$

Результирующие амплитуды (огибающие) сигналов в информационном и опорном отсчетах с учетом (16) и (17) будут, соответственно, равны:

$$
\begin{gathered}
A_{1}(0, \psi)=\sqrt{U_{1}^{2}(0)+U_{2}^{2}[-T(1-\chi)]+2 U_{1}(0) U_{2}[-T(1-\chi)] \cos \left(\psi-\varphi_{0}\right)} \\
A_{2}\left(\tau_{0}, \psi\right)=U_{1}\left(\tau_{0}\right) \sqrt{2\left[1+\cos \left(\psi-\varphi_{0}\right)\right]} .
\end{gathered}
$$

Вероятность ложной тревоги в первом канале обработки при некогерентном обнаружении определяется известным выражением [8]

$$
H_{1 F}=\exp \left(-\frac{\lambda^{2}}{2 P_{\mathrm{m}}}\right)
$$

где $\lambda$ - абсолютное значение порога принятия решения, а $P_{\text {шा }}$ - мощность шума на выходе согласованного фильтра.

При этом вероятность правильного обнаружения сигнала в первом канале обработки, выраженная через вероятность ложной тревоги с учетом (18), такова:

$$
H_{1 D}(\psi)=Q\left(\frac{A_{1}(0, \psi)}{\sqrt{P_{ш}}}, \sqrt{-2 \ln H_{1 F}}\right),
$$

где $Q(x, y)$ - функция Маркума.

Для получения вероятности ложной тревоги и правильного обнаружения во втором дополнительном канале обработки необходимо оперировать двумерной плотностью вероятности огибающих между информационным и опорным отсчетами при отсутствии и наличии навигационного сигнала.

Двумерная плотность вероятности огибающей в наших обозначениях при отсутствии сигнала имеет вид

$$
\Theta_{2}\left(U_{1}, U_{2}, \tau_{0}\right)=\frac{U_{1} U_{2}}{P_{\mathrm{II}}^{2}\left(1-R_{0}^{2}\right)} \exp \left(-\frac{U_{1}^{2}+U_{2}^{2}}{2 P_{\mathrm{II}}\left(1-R_{0}^{2}\right)}\right) I_{0}\left[\frac{R_{0} U_{1} U_{2}}{P_{\mathrm{II}}\left(1-R_{0}^{2}\right)}\right],
$$

где $I_{0}(\cdot)$ - функция Бесселя нулевого порядка, а $R_{0}=r\left(\tau_{0}\right)$ [5].

Одномерная плотность вероятности огибающей по опорному отсчету представляет собой распределение Релея [8]:

$$
\Theta\left(U_{2}\right)=\frac{U_{2}}{P_{\mathrm{I}}} \exp \left(-\frac{U_{2}^{2}}{2 P_{\mathrm{II}}}\right) .
$$

При этом условная плотность вероятности по информационному отсчету относительно опорного с учетом (22) и (23) будет равна

$$
\begin{gathered}
\Theta_{2}\left(U_{1}, \tau_{0} / U_{2}\right)=\frac{U_{1}}{P_{\mathrm{II}}\left(1-R_{0}^{2}\right)} \exp \left(-\frac{U_{1}^{2}+U_{2}^{2} R_{0}}{2 P_{\mathrm{II}}\left(1-R_{0}^{2}\right)}\right) I_{0}\left[\frac{R_{0} U_{1} U_{2}}{P_{\mathrm{II}}\left(1-R_{0}^{2}\right)}\right] . \\
-148-
\end{gathered}
$$


С учетом (24) выражение для вероятности ложной тревоги во втором канале обнаружения будет иметь вид

$$
H_{2 F}=Q\left[\frac{U_{\mathrm{\omega}}\left(\tau_{0}\right) R_{0}}{\sqrt{P_{\mathrm{\omega}}\left(1-R_{0}^{2}\right)}}, \frac{\lambda\left(\tau_{0}\right)}{\sqrt{P_{\mathrm{\omega}}\left(1-R_{0}^{2}\right)}}\right] .
$$

Если зафиксировать вероятность $H_{2 F}$, то из (25) можно получить выражение для управляемого порога во втором канале обработки:

$$
\lambda\left(\tau_{0}\right)=\sqrt{P_{\mathrm{w}}\left(1-R_{0}^{2}\right)}\left[Q^{-1}\left(H_{2 F}, \frac{U_{\mathrm{w}}\left(\tau_{0}\right) R_{0}}{\sqrt{P_{\mathrm{w}}\left(1-R_{0}^{2}\right)}}\right)\right],
$$

где $Q^{-1}(x, y)$ - функция, обратная функции Маркума.

В частном случае при $R_{0} \approx 0$ выражение (25) переходит в известное выражение (20) вероятности ложной тревоги.

При наличии навигационного сигнала плотность вероятности огибающих в информационном и опорном отсчетах будет иметь вид

$$
\begin{aligned}
& W_{2}\left(U_{1}, U_{2}, \tau_{0}\right)=\frac{U_{1} U_{2}}{P_{\mathrm{II}}^{2}\left(1-R_{0}^{2}\right)} \exp \left[-\frac{U_{1}^{2}+U_{2}^{2}+A_{1}^{2}+A_{2}^{2}-2 A_{1} A_{2} R_{0}}{2 P_{\mathrm{II}}\left(1-R_{0}^{2}\right)}\right] \times \\
& \times \sum_{n=0}^{\infty} \varepsilon_{n} I_{n}\left[\frac{R_{0} U_{1} U_{2}}{P_{\mathrm{II}}\left(1-R_{0}^{2}\right)}\right] I_{n}\left[\frac{A_{1}-R_{0} A_{2}}{P_{\mathrm{II}}\left(1-R_{0}^{2}\right)} U_{1}\right] I_{n}\left[\frac{A_{2}-R_{0} A_{1}}{P_{\mathrm{II}}\left(1-R_{0}^{2}\right)} U_{2}\right],
\end{aligned}
$$

где $I_{n}(\cdot)$ - функция Бесселя $n$-го порядка; $\varepsilon_{n}=1$ при $n=0$ и $\varepsilon_{n}=2$ при $n>0$ [5].

Плотность вероятности огибающей по опорному отсчету будет равна

$$
W\left(U_{2}\right)=\frac{U_{2}}{P_{\mathrm{w}}} \exp \left(-\frac{U_{2}^{2}+A_{2}^{2}}{2 P_{\mathrm{II}}}\right) I_{0}\left(\frac{U_{2} A_{2}}{P_{\mathrm{II}}}\right) .
$$

Условную плотность вероятности по навигационному отсчету относительно опорного определим, пользуясь (27) и (28):

$$
\begin{aligned}
& W_{2}\left(U_{1}, \tau_{0} / U_{2}\right)=\frac{U_{1}}{I_{0}\left(\frac{U_{2} A_{2}}{P_{\mathrm{II}}}\right) P_{\mathrm{II}}\left(1-R_{0}^{2}\right)} \exp \left[-\frac{U_{1}^{2}+U_{2}^{2} R_{0}^{2}+A_{1}^{2}+A_{2}^{2}-2 A_{1} A_{2} R_{0}}{2 P_{\mathrm{II}}\left(1-R_{0}^{2}\right)}\right] \times \\
& \times \sum_{n=0}^{\infty} \varepsilon_{n} I_{n}\left[\frac{R_{0} U_{1} U_{2}}{P_{\mathrm{II}}\left(1-R_{0}^{2}\right)}\right] I_{n}\left[\frac{A_{1}-R_{0} A_{2}}{P_{\mathrm{II}}\left(1-R_{0}^{2}\right)} U_{1}\right] I_{n}\left[\frac{A_{2}-R_{0} A_{1}}{P_{\mathrm{II}}\left(1-R_{0}^{2}\right)} U_{2}\right] .
\end{aligned}
$$

При этом вероятность правильного обнаружения будет определяться решением следующего интеграла:

$$
H_{2 D}=\int_{\lambda\left(\tau_{0}\right)}^{\infty} W\left(U_{1}, \tau_{0} / U_{2}\right) d U_{1} .
$$

Для упрощения выражения, определяющего $H_{2 D}$, применяется следующий прием. Исследования показывают, что при изменении напряжения в опорном отсчете $U_{2}$ плотность вероят- 
ности (29) смещается по оси значений аргумента практически без деформации своей формы. Следовательно, значение $H_{2}$ при любом $U_{2}=U_{\text {ш }}\left(\tau_{0}\right)$ будет практически таким же, как и при $U_{2}=0$, при котором условная плотность вероятности (29) принимает вид

$$
W_{2}\left(U_{1}, \tau_{0} / U_{2}\right)=\frac{U_{1}}{P_{\mathrm{m}}\left(1-R_{0}^{2}\right)} \exp \left[-\frac{U_{1}^{2}+\left(A_{1}-R_{0} A_{2}\right)^{2}}{2 P_{\mathrm{m}}\left(1-R_{0}^{2}\right)}\right] I_{0}\left[\frac{A_{1}-R_{0} A_{2}}{P_{\mathrm{I}}\left(1-R_{0}^{2}\right)} U_{1}\right] .
$$

В этом случае вероятность правильного обнаружения сигнала в соответствии с (30) с учетом (31) будет равна

$$
H_{2 D}=Q\left[\frac{A_{1}-R_{0} A_{2}}{\sqrt{P_{\mathrm{w}}\left(1-R_{0}^{2}\right)}}, \frac{\lambda\left(\tau_{0}\right)}{\sqrt{P_{\mathrm{w}}\left(1-R_{0}^{2}\right)}}\right]
$$

При отсутствии перекрытия навигационного и мешающего сигналов $U_{2}[-T(1-\chi)]=0$ и $R_{0}=0$ и выражение (32) переходит в известное выражение для вероятности правильного некогерентного обнаружения радиосигнала [8]:

$$
H_{2 D}=Q\left[\frac{U_{1}(0)}{\sqrt{P_{\mathrm{m}}}}, \frac{\lambda}{\sqrt{P_{\mathrm{m}}}}\right]
$$

Таким образом, выражения (25) и (32), соответствующие общему случаю, являются новыми и получены в данной работе впервые.

С учетом (18), (19) и (26) вероятность правильного обнаружения сигнала во втором канале обработки, выраженная через вероятность ложной тревоги, будет равна

$$
H_{2 D}(\psi)=Q\left[\frac{A_{1}(0, \psi)-R_{0} A_{2}\left(\tau_{0}, \psi\right)}{\sqrt{P_{\amalg}\left(1-R_{0}^{2}\right)}}, \sqrt{-2 \ln H_{2 F}}\right]
$$

При получении (34) использовано равенство $Q(0, x)=\exp \left(-\frac{x^{2}}{2}\right)[5]$.

Результирующие вероятности правильного обнаружения и ложной тревоги будут определяться общими с когерентным обнаружением выражениями (13) и (15) соответственно. При этом средняя результирующая вероятность правильного обнаружения по всем возможным значениям фазы Ч будет определяться интегралом (14), который вычислялся численным методом.

\section{Результаты исследования}

Для получения количественных результатов при когерентном и некогерентном обнаружении в расчетах использовалась гауссовская форма навигационного и мешающего радиосигналов с относительным временным перекрытием $\chi=0,7$. Отношение сигнал-фоновый шум по мощности принималось равным $h^{2}=40$ с результирующей вероятностью ложной тревоги $P_{F \text { рез }}=H_{F \text { рез }}=10^{-3}$. При этом исходная вероятность правильного обнаружения сигнала при 
отсутствии мешающих отражений и когерентном обнаружении составила $P_{D \text { исх }}=0.9996$, а при некогерентном обнаружении $H_{D \text { исх }}=0.997$.

В условиях мешающих отражений при постоянной составляющей начальной фазе $\varphi_{0}=\omega_{0} t_{0}=0$ и характерных соотношениях начальных фаз между навигационным и мешающим сигналами, равных $\psi=(0 ; \pi / 2 ; \pi)$ радиан (рад) по формулам (4), (11), (13) для когерентного обнаружения и по формулам (21), (34), (13) для некогерентного обнаружения, рассчитаны и сведены в табл. 1 значения вероятностей правильного обнаружения сигнала в первом канале обработки $P_{1 D}(\psi), H_{1 D}(\psi)$, во втором канале $P_{2 D}(\psi), H_{2 D}(\psi)$ и результирующей вероятности $P_{D \text { рез }}(\psi), H_{D \text { рез }}(\psi)$.

Из таблицы следует, что с увеличением фазового сдвига между навигационным и мешающим сигналами как для когерентного, так и для некогерентного случая вероятность правильного обнаружения в первом канале обработки уменьшается, а во втором - увеличивается. При этом результирующая вероятность правильного обнаружения с изменением фазового сдвига изменяется незначительно и при заданных исходных данных практически не уменьшается ниже $P_{D \text { рез }}(\psi)=0.999$ при когерентном обнаружении и $H_{D \text { рез }}(\psi)=0.993$ при некогерентном обнаружении. При этом средняя вероятность правильного обнаружения, полученная путем усреднения результирующей вероятности по всем значениям случайной фазы $\psi$, в соответствии с (14) при когерентном обнаружении равна $\overline{P_{D}}=0.9996$, а при некогерентном обнаружении $\overline{H_{D}}=0.996$, что свидетельствует о высокой эффективности данного способа обнаружения сигналов.

Такое поведение вероятностных характеристик объясняется наличием в приемнике двух каналов обработки, которые взаимно дополняют друг друга по эффективности [11]. Если в одном канале условия обнаружения ухудшаются, то во втором канале в тот же момент времени условия обнаружения улучшаются и в результате показатели итогового (результирующего) обнаружения практически не ухудшаются.

Например, если мешающий радиоимпульс находится в фазе с навигационным радиоимпульсом, то амплитуда результирующего сигнала в момент его регистрации резко возрастает. При этом достоверность обнаружения по первому каналу увеличивается, а по второму каналу уменьшается за счет возникновения составляющей сигнала (подставки) в опорном отсчете. Если мешающий радиоимпульс находится в противофазе с навигационным, то, наоборот, условия обнаружения по основному каналу ухудшаются по причине уменьшения амплитуды

Таблица. Значения вероятностей правильного обнаружения сигнала

\begin{tabular}{|c|c|c|c|}
\hline Характеристика & \multicolumn{3}{|c|}{ Значение } \\
\hline$\psi$, рад & 0 & $\pi / 2$ & $\pi$ \\
\hline$P_{1 D}(\psi)$ & 0.99999 & 0.9994 & 0.65 \\
\hline$H_{1 D}(\psi)$ & 0.9992 & 0.991 & 0.37 \\
\hline$P_{2 D}(\psi)$ & 0.023 & 0.674 & 0.9985 \\
\hline$H_{2 D}(\psi)$ & 0.212 & 0.671 & 0.989 \\
\hline$P_{D \text { рез }}(\psi)$ & 0.99999 & 0.9997 & 0.9994 \\
\hline$H_{D \text { рез }}(\psi)$ & 0.9994 & 0.997 & 0.993 \\
\hline
\end{tabular}


результирующего сигнала в момент его отсчета, а по дополнительному каналу условия обнаружения улучшаются, так как при этом исчезнет или станет ничтожно малой составляющая сигнала (подставка) в опорном отсчете. При этом в опорном отсчете будет измеряться только шум, по значению которого оценивается значение шума в информационном отсчете, на основе чего устанавливается требуемый переменный порог для когерентного обнаружения в соответствии с выражением (12) и для некогерентного обнаружения в соответствии с выражением (26). При промежуточных фазовых соотношениях между навигационным и мешающим радиосигналами будут соответствующим образом перераспределяться значения вероятности правильного обнаружения в каналах обработки.

\section{Заключение}

В связи с повышением рабочих частот современных навигационных радиотехнических систем повышается интенсивность соответствующих мешающих отражений, поступающих в точку приема сигнала. Использование классических одноканальных схем оптимальной обработки сигналов в этих условиях приводит к существенным ошибкам в оценках параметров и координат объектов, а также к ошибкам при различении информационных символов.

В данной статье разработан способ и соответствующие модели комплексной (двухканальной) обработки навигационных сигналов, позволяющие значительно ослабить влияние мешающих отражений на основные характеристики их обнаружения без увеличения энергетических и частотно-временных ресурсов радиоканала. Получены выражения для инженерных расчетов основных вероятностных характеристик обнаружения сигнала для общего случая наличия в канале связи сигналов мешающих отражений и флюктуационного шума.

Полученные результаты могут быть использованы как при модернизации существующих, так и при создании перспективных навигационных радиотехнических систем, обладающих повышенной эффективностью функционирования в условиях сложной помеховой обстановки.

Исследование выполнено за счет гранта Российского научного фонда (проект № 16-1910089).

\section{Список литературы}

[1] Фатеев Ю.Л., Гарин Е.Н., Дмитриев Д.Д., Тяпкин В.Н. Применение фильтрации при измерении угловой ориентации вращающегося объекта. Радиотехника, 2013, 6, 46-49 [Fateev Yu.L., Garin E.N., Dmitriev D.D., Tyapkin V.N. Filtering in the measurement of the angular orientation of the rotating object. Radiotekhnika, 2013, 6, 46-49 (in Russian)].

[2] Шарфунова Т.Г., Тяпкин В.Н., Дмитриев Д.Д. Точность измерения навигационных параметров в навигационной аппаратуре потребителя спутниковой радионавигационной системы ГЛОНАСС, оснащенной антенной решеткой. Радиотехника, 2013, 6, 22-25. [Sharfunova T. G., Tyapkin V. N., Dmitriev D. D. Measurement accuracy of navigation parameters of the navigation equipment of the consumer satellite navigation system GLONASS, are equipped with antenna array. Radiotekhnika, 2013, 6, 22-25 (in Russian)]. 
[3] Фатеев Ю.Л, Дмитриев Д.Д., Тяпкин В.Н, Кремез Н.С. Фазовые измерения в угломерной аппаратуре ГЛОНACC/GPS без разрешения фазовой неоднозначности. Наукоемкие технолоzиu, 2014, 9, 16-19 [Fateev Yu. L, Dmitriev D. D., Tyapkin V. N., Kremez N.S. Phase measurements in azimuth the equipment GLONASS/GPS without initialization. Science capacious technologies, 2014, 9, 16-19 (in Russian)].

[4] Фатеев Ю.Л, Дмитриев Д.Д., Тяпкин В.Н, Гребенников А.В., Бондарев В.Н. Разрешение фазовой неоднозначности в угломерной навигационной аппаратуре ГЛОНАCC/GPS. Уcneхи современной радиоэлектроники, 2014, 5, 67-70 [Fateev Yu.L., Dmitriev D. D., Tyapkin V. N., Grebennikov V. A., Bondarev V. N. Resolution of phase ambiguity in angle-measuring navigation equipment GLONASS/GPS. Successes of modern radioelectronics, 2014, 5, $67-70$ (in Russian)].

[5] Fateev Yu.L., Dmitriev D.D., Tyapkin V.N., Garin E.N., Shaidurov V.V. The phase ambiguity resolution in the angle-measuring navigation equipment. AIP Conference Proceedings, 2014, 12, 12 14, doi: 10.1063/1.4893795.

[6] Тяпкин В.Н., Фатеев Ю.Л.,. Дмитриев Д.Д,. Коннов В.Г. Калибровка измерительного тракта для испытания навигационной аппаратуры потребителей спутниковых радионавигационных систем на помехоустойчивость. Вестник СибГАУ, 2012, 4 (44), 139-142 [Tyapkin V.N., Fateev Yu.L., Dmitriev D.D, Konnov V.G. Calibration of the measuring path for the testing of navigation equipment of consumers of satellite navigation systems to interference. Vestnik SibGAU, 2012, 4 (44), 139-142 (in Russian)].

[7] Sardon E., Rius A., Zarraoa N. Estimation of the transmitter and receiver differential biases and the ionospheric total electron content from Global Positioning System observations. Radio Science, 1994, 29(3), 577-586.

[8] Тихонов В.И. Оптимальный прием сигналов. М.: Радио и связь, 1983 [Tikhonov V.I. Optimal signal reception. M.: Radio and communication, 1983 (in Russian)].

[9] Вентцель Е.С. Теория вероятностей. М. : Наука, 1969 [Wentzel E.S. Probability theory. M. : Nauka, 1969 (in Russian)].

[10] Евстафиев А.Ф. Способ обнаружения импульсного радиосигнала на фоне мемающего радиоимпульса и белого шума. Патент на изобретение № 2160905. опубл. 20.12.2000; бюл. № 34 [Evstafiev A.F. Sposob obnaruzheniia impulsnogo radiosignala na fone meshaiushchego radioimpulsa i belogo shuma. Patent 2160905, ed. 20.12.2000, bul. 34].

[11] Евстафиев А.Ф. Способ некогерентного обнаружения импульсного радиосигнала на фоне мешающего радиоимпульса и белого шума. Патент на изобретение № 2285274. опубл. 10.10.2006; бюл. № 28 [Sposob nekogerentnogo obnaruzheniia impulsnogo radiosignala na fone meshaiushchego radioimpulsa i belogo shuma. Patent 2285274, ed. 10.10.2006, bul. 28]. 